\title{
Optimización de uso del suelo acorde con restricciones ambientales y sociales: caso cuenca de Riogrande $\mathrm{II}^{*}$
}

\author{
Recibido: 7 de febrero de 2019 • Aprobado: 23 de mayo de 2019 \\ https://doi.org/10.22395/seec.v22n52a2 \\ Juan David Osorio Múnera** \\ Natalia Uribe Rivera ${ }^{* * *}$ \\ Lina Marcela García-Tavera*** \\ Eduardo Andrés Bustamante Ochoa ${ }^{* * * *}$
}

\section{RESUMEN}

Las actividades productivas que se realizan en la cuenca del embalse de Riogrande II (Antioquia, Colombia) generan afectaciones ambientales negativas sobre suelo y agua. La integración de modelos hidrológicos y económicos provee una base científica para un escenario planeado de uso del territorio, que sea racional y adecuado a la actual vocación agropecuaria. Partiendo de los datos generados a partir del modelo SWAT se reformula el modelo Ecosaut para determinar el uso óptimo de suelo acorde con restricciones ambientales y sociales, encontrando que, para asegurar la sostenibilidad de la cuenca, es necesario implementar un sistema de rotación compuesto por pastos y cultivo de papa junto con una reducción de alrededor del 42 \% del suelo actualmente dedicado a actividades agrícolas.

\section{PALABRAS CLAVE}

Uso de suelo; optimización; cuencas hidrográficas; SWAT; Ecosaut.

JEL

Q12, Q15, Q24, Q25.

\section{CONTENIDO}

Introducción; 1. Caracterización de la cuenca; 2. Metodología; 3. Resultados y discusión; 5. Conclusiones; Bibliografía.

- Artículo de investigación, grupo Microeconomía Aplicada, Universidad de Antioquia, clasificación A2, producto del proyecto Efectos económicos y ambientales de los usos del suelo: bases para una política ambiental de incentivos en sistemas productivos agroindustriales, financiado por Colciencias 2014-2018.

* Economista, Universidad de Medellín, Medellín, Colombia. Magíster en Economía de los Recursos Naturales y del Medio Ambiente, Universidad de los Andes, Bogotá, Colombia. Docente, Departamento de Economía, Universidad de Antioquia, Medellín, Colombia. Integrante grupo de Microeconomía Aplicada, Universidad de Antioquia. Medellín, Colombia. Correo electrónico: juan.osorio35@udea. edu.co

** Ingeniera topográfica, Universidad del Valle, Cali, Colombia. Master of Science in Enviromental Science, Unesco-IHE, Delft, Holanda. Máster en Ingeniería, Universidad del Valle, Cali, Colombia. Ph. D. Research Fellow in Hydroinformatics, TU Delft and IHE-Delft, Delft, Holanda. Correo electrónico: n.uriberivera@un-ihe.org

*** Abogada, Universidad de Antioquia, Medellín, Colombia. Estudiante de Maestría en Economía, Universidad de Antioquia, Medellín, Colombia. Estudiante de Doctorado en Economía, Universidad del Rosario, Bogotá, Colombia. Integrante grupo de Microeconomía Aplicada, Universidad de Antioquia, Bogotá, Colombia. Correo electrónico: lina.garciat@udea.edu.co

..** Economista, Universidad de Antioquia, Medellín, Colombia. Estudiante de la Maestría en Economía, Universidad de Antioquia, Medellín, Colombia. Estudiante asistente del grupo de Microeconomía Aplicada, Universidad de Antioquia, Medellín, Colombia. Correo electrónico: eandres.bustamante@ udea.edu.co 


\title{
OPTIMIZATION OF SOIL USE ACCORDING TO THE ENVIRONMENTAL AND SOCIAL RESTRICTIONS: THE CASE OF THE RIOGRANDE II BASIN
}

\begin{abstract}
The productive activities carried out at the basin of the Riogrande II press (Antioquia, Colombia) generate negative environmental affections upon the soil and water. The integration of hydrological and economical models provides a scientific foundation for planned use of the territory that is rational and adequate for the actual agricultural vocation. Starting from the data generated by the SWAT model, the Ecossaut model is reformulated for determining the optimal use of the soil according to the social and environmental restrictions, finding out that in order to reassure the reliability of the basin is necessary an implementation of rotation system composed of grass and potato crops along with a reduction of around $42 \%$ of the soil currently used for agricultural activities.
\end{abstract}

\section{KEYWORDS :}

Use of soil; optimization; hydrographic basins; SWAT; Ecosaut.

JEL CODE

Q12, Q15, Q24, Q25.

\section{CONTENTS}

Introduction; 1. Basin characterization; 2. Methodology; 3. Results and discussion; 5. Conclusions; Bibliography

\section{OTIMIZAÇÃO DO USO DO SOLO CONFORME RESTRIÇÕES AMBIENTAIS E SOCIAIS: CASO BACIA DE RIOGRANDE ॥}

\section{RESUMO}

As atividades produtivas realizadas na bacia de Riogrande II (Antioquia, Colômbia) geram afetações ambientais negativas sobre o solo e a água. A integração de modelos hidrológicos e econômicos fornece uma base científica para um cenário planejado de uso do território que seja racional e adequado à agropecuária. A partir de dados gerados com o modelo SWAT, o modelo Ecosaut é reformulado para determinar o ótimo uso do solo de acordo com restrições ambientais e sociais; assim, verificou-se que, para garantir a sustentabilidade da bacia, é necessário implantar um sistema de rodízio de pastagem e de plantação de batata junto com uma redução de 42 \% do solo atualmente dedicado a atividades agrícolas.

\section{PALAVRAS-CHAVE}

Uso de solo; otimização; bacias hidrográficas; SWAT; Ecosaut.

JEL

Q12, Q15, Q24, Q25.

\section{CONTEÚDO}

Introdução; 1. Caracterização da bacia; 2. Metodologia; 3. Resultados e discussão; 5 . Conclusões; Bibliografia 


\section{INTRODUCCIÓN}

Los ecosistemas andinos son altamente sensibles a la degradación ambiental. De acuerdo con Cárdenas et al. (2009), las condiciones climáticas, una geografía de altas pendientes y los procesos productivos en la región aumentan la vulnerabilidad y ponen en riesgo el potencial hidrológico y la calidad del suelo. El proyecto Efectos económicos y ambientales de los usos del suelo: bases para una política ambiental de incentivos en sistemas productivos agroindustriales estudia los sistemas de producción que se desarrollan en la cuenca que alimenta el embalse de Riogrande II y establece relaciones entre estas actividades económicas y los recursos hídricos para determinar usos de suelo óptimos.

El embalse Riogrande II se encuentra ubicado en la región norte del departamento de Antioquia, Colombia (1.000 a 4.000 MSNM), es una de las principales fuentes de agua potable del Valle de Aburrá, segundo centro urbano del país. Hay predominio de parcelas subfamiliares con fines de explotación ganadera y siembra de cultivos de subsistencia, generalmente con prácticas inadecuadas y de baja tecnología que dejan descubierto el suelo, alteran los componentes físicos y químicos con el arrastre de partículas que terminan por aumentar los niveles de erosión y sedimentación de las corrientes de agua, según estudio realizado por Corantioquia y UNAL (2012). Como señala Cárdenas (2013), estudios de campo evidencian las dificultades que enfrentan entidades públicas y privadas para implementar medidas de protección de los recursos naturales en la zona, lo que indica una gestión ambiental costosa, ineficiente y difícil de mantener.

Parte del trabajo empírico en torno a políticas para el control de impactos de la agricultura sobre agua y suelo ha combinado modelos de producción agrícola en múltiples escalas con modelos hidrológicos para analizar los efectos de políticas y para determinar cuáles estrategias minimizan costos y permiten alcanzar una calidad ambiental determinada. Entre los modelos hidrológicos se destaca el modelo de simulación SWAT (Soil and Water Assessment Tool) que permite analizar los efectos sobre los suelos de las actividades agroindustriales y entender la hidrología de la cuenca. Como lo muestran Yevenes y Mannaerts (2011), y Lam, Schmalz y Fohrer (2011), los resultados del modelo tienen amplia aceptación en la evaluación de los impactos de cambio en el uso de la cobertura del suelo para diferentes escenarios. Además, como agregan Kok, Verburg y Velkamp (2004), tiene un alcance más profundo por simular la dinámica del paisaje en el futuro a escalas múltiples y diferenciadas.

La integración del modelo SWAT con modelos económicos de uso del suelo permite un diagnóstico más exacto, además, mejora la comprensión de procesos 
clave del territorio, según lo reseñan Lambin, Rounsevell, y Geist (2000). El modelo Ecosaut, formulado por Quintero, Estrada y García (2006), permite simular y cuantificar los efectos del uso del suelo a medida que varían los niveles de nitratos y fosfatos. Este modelo fue diseñado teniendo presentes las particularidades de las cuencas ubicadas en la región de los Andes americanos y varias aplicaciones han demostrado su utilidad como herramienta para la planificación social. Particularmente, Quintero, Wunder y Estrada (2009) analizan para dos cuencas de Ecuador y Perú en el contexto de esquemas de pagos por servicios ambientales (PES por sus siglas en inglés) los beneficios económicos de los proveedores de estos servicios. Un trabajo similar realizan León y Renner (2010) para Perú usando Ecosaut como herramienta para el cálculo de costos y beneficios asociados a diferentes usos de tierra. Las aplicaciones han estado enfocadas al modelo de los PES.

Aunque la estructura básica del modelo Ecosaut original se mantiene, para obtener los resultados de este estudio se acondicionaron los resultados promedio del modelo SWAT por URH (Unidad de Respuesta Hidrológica) y por latitud de la cuenca en forma agregada como parte funcional e integrada al proceso de optimización del uso del suelo ante las distintas alternativas de rotación de cultivos. Las series de precios de leche, carne, salarios agropecuarios y concentrados animales, fueron corregidas por inflación usando como deflactores el índice de precios al productor (IPP) con base en 2015 para la agricultura, la ganadería, la caza, la silvicultura y la pesca; y el IPP de los salarios de la industria manufacturera del Banco de la República. Desde el punto de vista operacional, un aporte importante del nuevo modelo es la inclusión de las alternativas típicas de rotación de cultivos de la zona de la cuenca de Riogrande II, los cuales alternan el uso de suelo entre los cultivos de papa capira, tomate de árbol y pastos para la producción de leche. Asimismo, el flujo de ingresos netos del productor agropecuario fue calculado en términos del valor presente neto (VPN) asociado a la tasa de descuento promedio de la DTF otorgada por el mercado financiero nacional.

El objetivo es mostrar, a partir de la integración de metodologías de corte hidrológico con modelos económicos de optimización, el uso óptimo de suelo que garantice la sostenibilidad de la zona de la cuenca cumpliendo unas restricciones sociales y ambientales. La estructura del texto es la siguiente: i) caracterización de la cuenca, ii) descripción de las metodologías, iii) resultados y discusión, iv) conclusiones. Se concluye que para asegurar la sostenibilidad de la cuenca es necesario implementar un sistema de rotación compuesto por pastos y cultivos de papa junto con una reducción de alrededor del 42 \% del suelo actualmente dedicado a actividades agrícolas. 


\section{CARACTERIZACIÓN DE LA CUENCA}

La principal actividad agropecuaria en los municipios de la cuenca del embalse de Riogrande II es la ganadería de leche especializada, seguida de cultivos (especialmente papa y tomate de árbol) y otras actividades en menor escala como la porcicultura (ver gráfico 1). Estos sistemas productivos no cuentan con niveles significativos de tecnología aplicada a los procesos de producción lo que ha contribuido con la degradación y empobrecimiento de los suelos.

Gráfico 1. Usos del suelo en pastos manejados, tomate de árbol, papa y otros cultivos transitorios

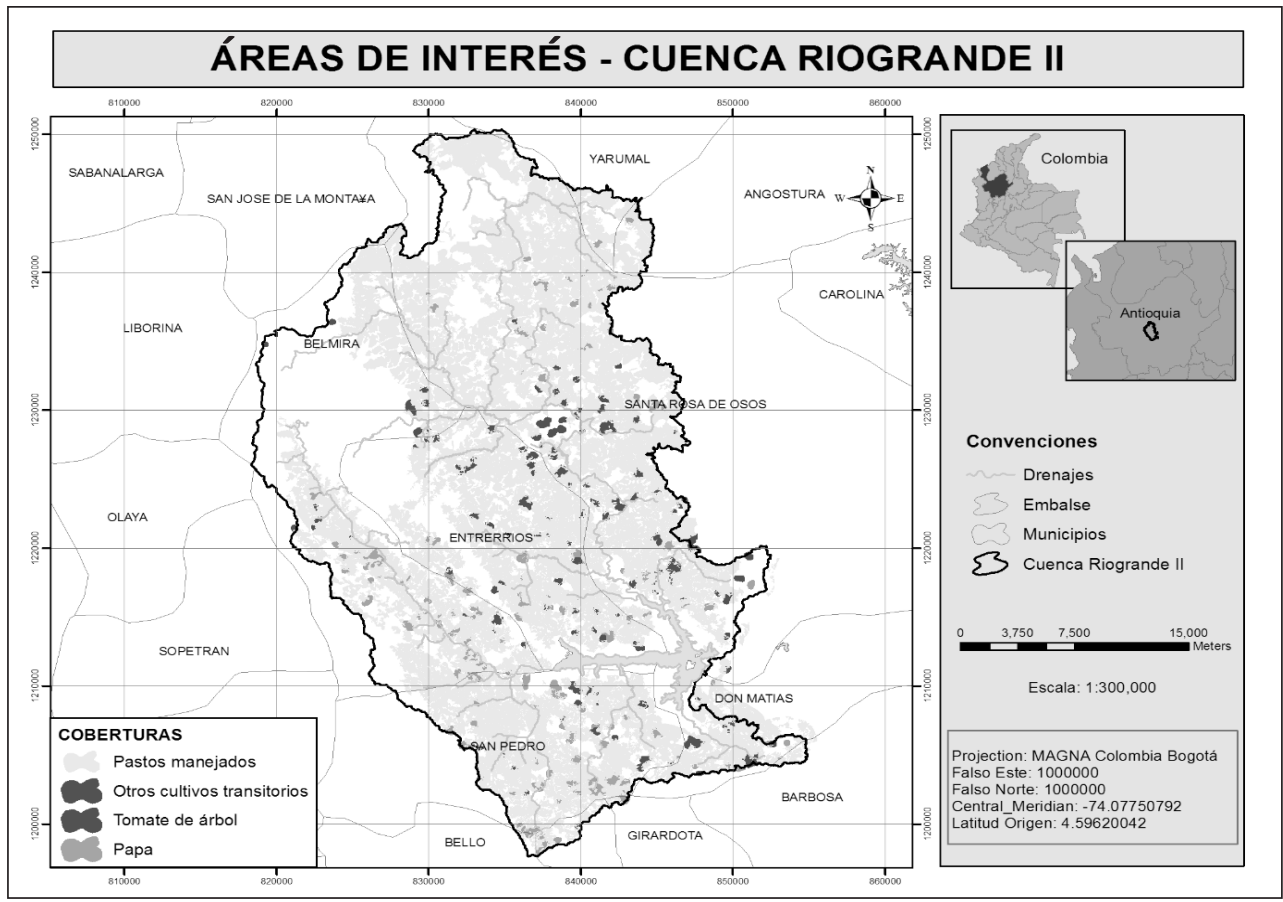

Fuente: Utgra (2018).

\subsection{Producción lechera}

Utilizando resultados de encuestas realizadas a 44 unidades productivas o fincas especializadas en leche ubicadas en la zona de influencia de la cuenca, se estimaron valores promedio de costos, prácticas de manejo y capacidad productiva para extraer datos finales de la unidad productiva representativa utilizada en el modelo. Se trabajó mediante una encuesta inicial sobre características generales y luego se realizó un monitoreo, durante algunos meses, sobre parámetros productivos y reproductivos manejados en cada una de las fincas. 
Los resultados evidencian que, en promedio, el uso predominante en la zona es alrededor de 19,1 ha. Las fincas tienen capacidad de albergar entre 30 y 50 vacas lecheras, con un promedio de 36 vacas/finca. Los pastos usados son las variedades kikuyo (Pennisetun clandeslinum), ryegrass (Lolium multiflorum) y falsa poa (Holcus lanalus). La variedad kikuyo es más usada debido a su gran resistencia y capacidad de rebrote, por lo que se asumirá como predominante para el territorio. $100 \%$ de las fincas utilizan fertilizante químico y alrededor del 70 \% lo combinan con porquinaza. Bajo un periodo de rotación promedio de 38 días, se estima que cada finca aplica 1,75 t de nitratos y 1,25 t de fosfatos para mantenimiento de pastos.

Mayoritariamente, son pequeños productores con un promedio de 17 litros/día de leche por vaca. Alrededor del 80 \% de los productores encuestados le venden su producción a la empresa Colanta a un precio promedio de \$979 por litro. La mayoría de mano de obra utilizada en el ordeño es masculina y familiar a un precio promedio de \$29.656 el jornal, mientras que la mano de obra contratada externa se paga alrededor de \$29.904. El modelo económico no tiene en cuenta los costos de inversión iniciales necesarios para costear las instalaciones, se asume que ya estaban establecidas al inicio del periodo.

\subsubsection{Costos manejo de pastos}

El proceso completo de siembra y rotación durante el año se presenta en el gráfico 2, que muestra alternancia entre fertilización química y fertilización orgánica con porquinaza para 1,5 y 2 meses por cada periodo de pastoreo.

Gráfico 2. Esquema de siembra y fertilización de pastos bajo sistema de riego con porquinaza

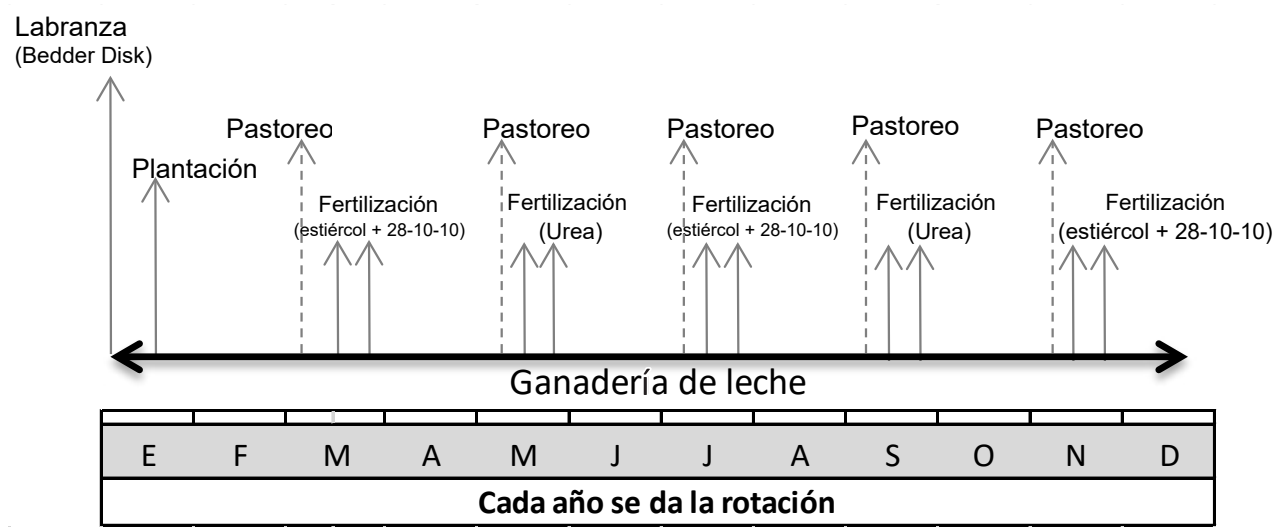

Fuente: Utgra (2018). 
Los resultados de rendimiento y eficiencia en el aprovechamiento del forraje de los pastos se utilizan para calcular los niveles de energía y proteína necesarios para el sostenimiento del ganado.

Tabla 1. Costos del manejo de pastos

\begin{tabular}{|c|c|}
\hline Variables estimadas & Costo por hectárea \\
\hline Fertilización & $\$ 126.940$ \\
\hline Plaguicidas & $\$ 13.177$ \\
\hline Costo de mano de obra & $\$ 16.399$ \\
\hline Costo total manejo de potreros & $\$ 172.036$ \\
\hline Costo por kg de forraje verde $\left(\$ / \mathrm{m}^{2}\right)$ & $\$ 22$ \\
\hline Costo total por periodo de rotación (38 días) & $\$ 183.166$ \\
\hline Desperdicio $\left(\mathrm{kg} / \mathrm{m}^{2}\right)$ & 0,89 \\
\hline Productividad neta (biomasa aprovechada) $\left(\mathrm{kg} / \mathrm{m}^{2}\right)$ & 1,20 \\
\hline Productividad bruta $\left(\mathrm{kg} / \mathrm{m}^{2}\right)$ & 2,08 \\
\hline Pasto aprovechado por animales en X m²/rotación & $248.838,91$ \\
\hline
\end{tabular}

Fuente: Utgra (2018).

\subsubsection{Costos del manejo de terneras y novillas}

Aunque muchos productores prefieren comprar terneras en plena edad reproductiva, en el modelo económico se asume que pueden criar terneras o especializarse únicamente en la producción de leche.

Tabla 2. Costos del levante promedio de terneras

Variables estimadas

Costo total de levante (\$/animal)

Valor promedio por $\mathrm{kg}$ de concentrado levante $(\$ / \mathrm{kg})$

Costo total de concentrado levante (\$)

Costo total de forraje (pasto verde) levante (\$)

Leche o lactoreemplazador hasta el destete

Sales, sodas, heno y suplementos dietarios

Costo de medicamentos, higiene, desinfección y salud animal
Costo de levante de terneras por periodo de rotación promedio

$\$ 1.172 .843$

$\$ 1.017$

$\$ 212.206$

$\$ 291.907$

$\$ 376.437$

$\$ 43.302$

$\$ 47.310$ 
Juan David Osorio Múnera, Natalia Uribe Rivera, Lina Marcela García-Tavera y Eduardo Andrés Bustamante Ochoa

Variables estimadas

Costo de levante de terneras por periodo de rotación promedio

\begin{tabular}{ll}
\hline Costo en insecticidas y garrapaticidas & $\$ 12.760$ \\
\hline Mano de obra (cuidado) & $\$ 86.313$ \\
\hline Asistencia profesional (veterinario) & $\$ 17.064$ \\
\hline Total & $\$ 2.261 .159$ \\
\hline
\end{tabular}

Fuente: Utgra (2018).

En las fincas que optan por criar terneras o novillas, la gran mayoría de los terneros que nacen son descartados en la primera semana de edad y vendidos a un precio aproximado de $\$ 70.000$ por animal, dependiendo del peso del ternero, que en promedio es $43 \mathrm{~kg}$ para la raza Holstein, esto de acuerdo con lo reportado por Corantioquia (2015).

\subsubsection{Costos de alimentación, ordeño y mantenimiento de ganado de producción}

Los costos corresponden a la estimación promedio de un hato ganadero especializado en leche con capacidad media de 36 vacas que producen alrededor de 634 litros/día.

Tabla 3. Costos del manejo de ganado en producción de leche

\begin{tabular}{lc}
\hline \multicolumn{1}{c}{ Variables estimadas } & $\begin{array}{c}\text { Costo promedio de alimentación, } \\
\text { ordeño y manutención }\end{array}$ \\
\hline Costos de mano de obra $(\$)$ & $\$ 1.544 .231$ \\
\hline Costo total de concentrado de producción $(\$)$ & $\$ 3.768 .947$ \\
\hline Sales, sodas y suplementos dietarios & $\$ 134.954$ \\
\hline Costo de medicamentos, higiene, desinfección y salud animal & $\$ 577.190$ \\
\hline Costo total de forraje (pasto verde) en producción $(\$)$ & $\$ 1.035 .723$ \\
\hline Costo total promedio (\$ hato/mes) & $\$ 9.125 .410$ \\
\hline Costo total promedio (\$ hato/periodo de rotación) & $\$ 10.877 .941$ \\
\hline
\end{tabular}

Fuente: Utgra (2018).

\subsection{Principales cultivos transitorios}

Según el Pomca (Plan de Manejo y Ordenamiento de una Cuenca) de Corantioquia (2015) el principal cultivo transitorio de la zona es la papa, que se realiza temporalmente y como actividad secundaria para renovación de potreros. Se caracteriza 
por el uso intensivo de fertilizantes, plaguicidas y maquinaria para asegurar altos rendimientos. El cultivo de papa se toma siempre como transitorio en todos los predios, llevado a cabo cada tres años de forma intermitente (gráfico 3). Los productores encuestados argumentan que de un terreno solo se pueden sacar dos o tres cosechas de buena calidad, lo que hace que el cultivo resulte inviable económicamente de forma permanente.

Gráfico 3. Esquema de siembra y fertilización de papa bajo sistema de rotación intermitente cada tres años

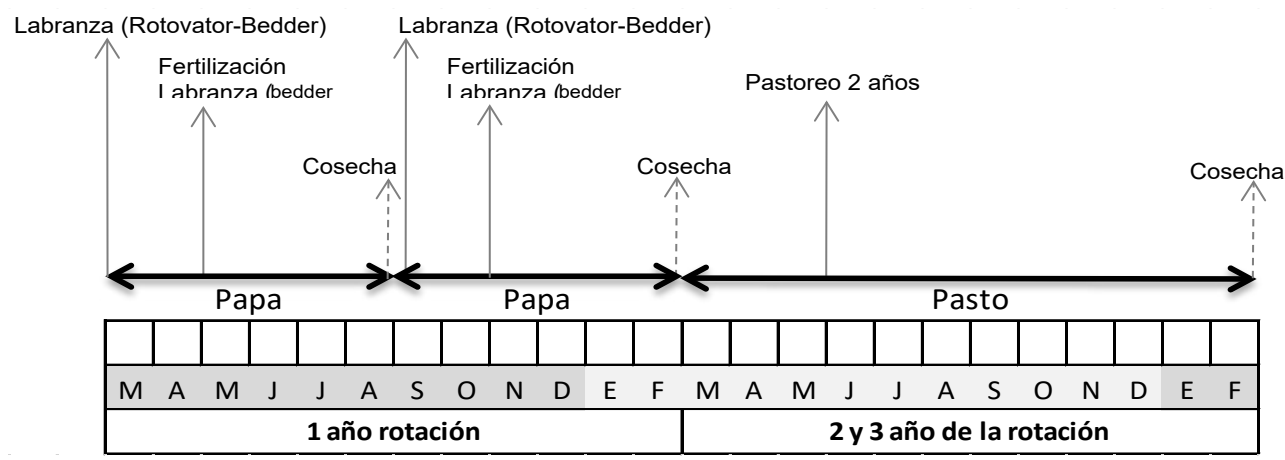

Fuente: Utgra (2018).

La información fue obtenida de un productor de capacidad media en la zona, que se dedica expresamente al cultivo de papa bajo una modalidad preponderante en la región: alquila o acuerda el uso del suelo con los propietarios para la producción de papa por un año, posteriormente, deja adecuados los terrenos para cultivos de pastos.

Tabla 4. Costos del manejo del cultivo de papa anuales por hectárea

\begin{tabular}{lc}
\hline \multicolumn{1}{c}{ Actividades para la siembra de papa (productor capacidad media) } & $\begin{array}{c}\text { Costo anual por hectárea } \\
\text { (2 periodos de rotación) }\end{array}$ \\
\hline Semilla y desacidización de la tierra & $\$ 3.256 .100$ \\
\hline Abono orgánico (siembra) & $\$ 175.500$ \\
\hline Abono químico (siembra) & $\$ 82.350$ \\
\hline Abono químico (aporque) & $\$ 146.290$ \\
\hline Insecticidas y control de plagas & $\$ 74.300$ \\
\hline Fungicidas y herbicidas & $\$ 45.705$ \\
\hline Coadyuvantes & $\$ 3.850$ \\
\hline Empaques & $\$ 12.000$ \\
\hline
\end{tabular}


Juan David Osorio Múnera, Natalia Uribe Rivera, Lina Marcela García-Tavera y Eduardo Andrés Bustamante Ochoa

\begin{tabular}{lc}
\hline \multicolumn{1}{c}{ Actividades para la siembra de papa (productor capacidad media) } & $\begin{array}{c}\text { Costo anual por hectárea } \\
\text { (2 periodos de rotación) }\end{array}$ \\
\hline Maquinaria & $\$ 320.000$ \\
\hline Mano de obra & $\$ 9.018 .000$ \\
\hline Transporte & $\$ 508.000$ \\
\hline Administración & $\$ 50.000$ \\
\hline Costos totales & $\$ 13.692 .095$ \\
\hline
\end{tabular}

Fuente: Utgra (2018).

\subsection{Principales cultivos semipermanentes y permanentes}

El cultivo semipermanente del tomate de árbol es el de mayor importancia. Luego de tres años, el ciclo de vida de la planta culmina, por lo que el tomate generalmente es utilizado como cultivo precursor de pastos. Incluso los periodos de cultivo no alcanzan los tres años debido a que su productividad cae aceleradamente con el avance del ciclo de vida y con el aumento de la probabilidad de enfermedades y hongos.

El cultivo de tomate de árbol es exigente en humedad y fertilizaciones. Generalmente se utilizan fertilizantes químicos con altos porcentajes de nitrógeno (N), fósforo (P) y potasio (K). Se aplican de 200 a 300 gramos de producto comercial por árbol cada cuatro meses, se inicia ocho meses después de siembra y se aumenta la dosis con el crecimiento del árbol.

Gráfico 4. Esquema de siembra y fertilización de tomate de árbol bajo sistema de cultivo semipermanente con renovación cada tres años

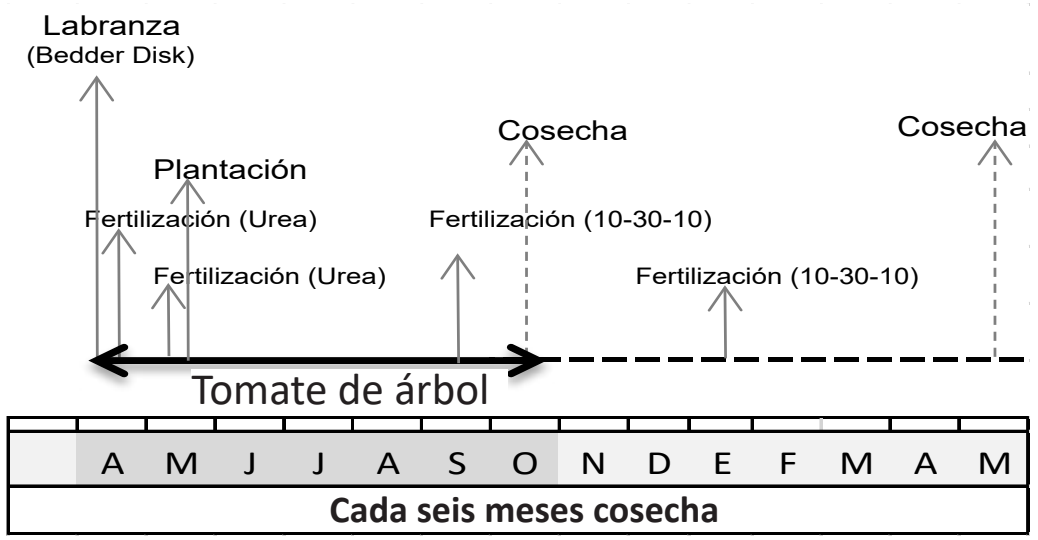

Fuente: Utgra (2018). 
La tabla 5 resume los gastos de un productor de capacidad media a lo largo de tres años, los datos corresponden a las estimaciones de los costos anuales promedio por hectárea de un proyecto con capacidad media de producción como cultivo precursor de pastos.

Tabla 5. Costos del manejo del cultivo de tomate de árbol por hectárea

\begin{tabular}{|c|c|}
\hline Actividades para la siembra de tomate de árbol (productor capacidad media) & Costo anual por hectárea \\
\hline Costos de mano de obra & $\$ 3.050 .000$ \\
\hline Fertilizantes químicos & $\$ 6.700 .933$ \\
\hline Fertilizante orgánico & $\$ 2.460 .000$ \\
\hline Insecticidas y fungicidas & $\$ 1.015 .133$ \\
\hline Otros insumos & $\$ 1.479 .500$ \\
\hline Costos totales por hectárea & $\$ 39.615 .333$ \\
\hline Costos totales sin mano de obra & $\$ 36.565 .333$ \\
\hline
\end{tabular}

Fuente: Utgra (2018).

\section{METODOLOGÍA}

\subsection{Modelo hidrológico}

El modelo SWAT, desarrollado por el Departamento de Agricultura de los Estados Unidos -Servicio de Investigación Agrícola (USDA-ARS) - es un modelo de cuencas hidrográficas semidistribuido, en tiempo continuo y basado en procesos, diseñado para simular los efectos de las decisiones de gestión en la cantidad y calidad del agua, definición de Neitsch, Arnold y Williams (2011). Siguiendo a Arnold et al. (1998), las unidades de procesamiento del modelo son unidades de respuesta hidrológica (UHR): pequeñas áreas de tierra con combinaciones únicas de tipo de suelo, uso del suelo y prácticas de manejo agrícola. El ciclo hidrológico se basa en la ecuación de balance hídrico:

$$
\mathrm{SW}_{t}=\mathrm{SW}_{0}+\sum_{i=1}^{t}\left(\mathrm{R}_{\text {day }}-\mathrm{O}_{\text {surf }}-\mathrm{E}_{a}-\mathrm{W}_{\text {seep }}-\mathrm{Q}_{q w}\right)
$$

Fuente: adaptado de Arnold et al. (1998).

Donde, $t=$ tiempo (día); $\mathrm{SW}_{t}=$ contenido final de agua en el suelo ( $\mathrm{mm}$ de agua); $\mathrm{SW}_{0}=$ contenido de agua inicial en el día $i(\mathrm{mmH} 2 \mathrm{O}) ; \mathrm{R}_{\text {day }}=$ cantidad de precipitación en el día $i(\mathrm{mmH} 2 \mathrm{O}) ; \mathrm{W}_{\text {seep }}=$ cantidad de agua acumulada en la zona no saturada del perfil del suelo en día $i(\mathrm{mmH2O}) ; \mathrm{y}_{\mathrm{qw}}=$ cantidad de flujo de retorno en el día $i$ (mm de agua). 
Fueron utilizadas las siguientes ecuaciones al representar la cuenca hidrográfica de Riogrande II. Para predecir la escorrentía superficial, se utilizó el método del número de curva (CN) del Servicio de Conservación de los Recursos Naturales (USDA-SCS, 1972). Siguiendo a Neitsch, Arnold y Williams (2011), las estimaciones del modelo de las cantidades de escorrentía según el uso de la tierra y los tipos de suelo varían así:

$$
\mathrm{Q}_{\text {surf }}=\frac{\left(\mathrm{R}_{\text {day }}-\mathrm{I}_{a}\right)^{2}}{\left(\mathrm{R}_{\text {day }}-\mathrm{I}_{a}+\mathrm{S}\right)}
$$

Donde, Qsurf = escorrentía acumulada o exceso de lluvia (mm H2O); Rday = profundidad de precipitación ( $\mathrm{mm} \mathrm{H2O}$ ); Ia = abstracción inicial, que incluye el almacenamiento en la superficie, así como la intercepción y la infiltración antes de la escorrentía (mm H2O). La abstracción inicial se suele aproximar a 0,2S, y S es el parámetro de retención (mm H2O). En esta abstracción, S = 25,4 * [(1000/CN) - 10] y CN es el número de curva para el día. El CN es una función del uso de la tierra, la permeabilidad del suelo y las condiciones anteriores del agua del suelo. Se utilizaron dos métodos para calcular el parámetro de retención: i) el método de humedad del suelo, que permite que el parámetro de retención varíe con el contenido de agua del perfil del suelo y ii) el método ET de la planta, que permite que el parámetro de retención varíe con la evapotranspiración acumulada de la planta.

El nitrato- $\mathrm{N}$ se puede transportar con escorrentía superficial, percolación y flujo lateral. La concentración de nitrato- $\mathrm{N}$ en el agua móvil debe calcularse para determinar la cantidad de nitrato-N movida por el agua. La cantidad de agua móvil se refiere a la cantidad de agua perdida por escorrentía superficial $\left(\mathrm{Q}_{\text {surf }}\right)$, flujo lateral $\left(\mathrm{Q}_{\text {lat }, l y)}\right.$ y percolación $\left(w_{\text {perc }, l y}\right)$.

$$
\operatorname{conc}_{\mathrm{NO} 3, \text { mobile }}=\frac{\mathrm{NO}_{l y}\left[1-\exp \left(\frac{-w_{\text {mobile }}}{\left(1-\theta_{e}\right) \cdot \mathrm{SAT}_{l y}}\right)\right]}{w_{\text {mobile }}}
$$

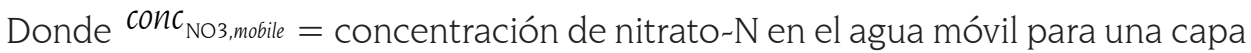
dada $\left(\mathrm{kg} \mathrm{N} / \mathrm{mm} \mathrm{H}_{2} \mathrm{O}\right.$ ), ), $\mathrm{NO}_{l y}$ = cantidad de nitrato-N en la capa (g N/ha ), $\theta \mathrm{e}=$ fracción de porosidad, $\mathrm{SAT}_{l y}=$ contenido de agua saturada de la capa del suelo ( $\mathrm{mm}$

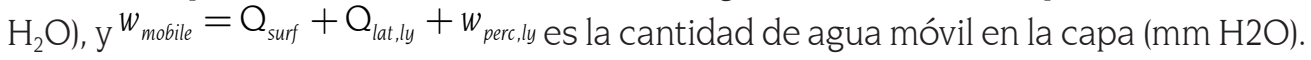
La siguiente ecuación calcula el nitrato-N eliminado en la escorrentía superficial:

$$
\mathrm{NO}_{\text {surf }}=\beta_{\mathrm{NO} 3} \cdot \operatorname{conc}_{\mathrm{NO} 3, \text { mobile }} \cdot \mathrm{Q}_{\text {surf }}
$$


La movilidad de la solución de fósforo es baja, por lo tanto, en el modelo SWAT, la escorrentía superficial solo interactúa parcialmente con la solución P almacenada en los $10 \mathrm{~mm}$ superiores del suelo.

$$
\mathrm{P}_{\text {surf }}=\frac{\mathrm{P}_{\text {solutionsurf }} \cdot \mathrm{Q}_{\text {surf }}}{\rho_{b} \cdot \operatorname{depth} h_{\text {surf }} \cdot k_{d, \text { surf }}}
$$

Donde, $\mathrm{P}_{\text {solutionsurf }}=$ cantidad de fósforo en solución en los $10 \mathrm{~mm}$ superiores ( $\mathrm{kg} \mathrm{P} / \mathrm{ha}) ; \rho_{6}$ es la densidad aparente de los $10 \mathrm{~mm}$ superiores $\left(\mathrm{Mg} / \mathrm{m}^{3}\right)$; depth $h_{\text {surf }}=$ profundidad de la capa superficial $(10 \mathrm{~mm})$ y $k_{d, \text { surf }}=$ coeficiente de partición del suelo de fósforo $\left(\mathrm{m}^{3} / \mathrm{Mg}\right)$. Esto se refiere a la proporción de la concentración de fósforo soluble en los $10 \mathrm{~mm}$ superiores del suelo.

\subsubsection{Datos de entrada y configuración del modelo}

Los insumos necesarios para el modelo incluyen información espacial, como el uso de la tierra, el tipo de suelo, la topografía, el clima y las prácticas de manejo agrícola (cronogramas de manejo). Los registros diarios de precipitación y temperatura (máximo, mínimo y promedio), así como los registros mensuales de humedad relativa, radiación solar y velocidad del viento se obtuvieron de las estaciones ubicadas en la cuenca de las Empresas Públicas de Medellín -EPM (2005) (gráfico 5a). El modelo digital de elevación a una resolución espacial de 12,5 m, fue elaborado con base en la topografía de curvas de nivel con equidistancia de 10 m realizado por la UNAL y Corantioquia (gráfico 5b). Se utilizó el mapa de uso del suelo del Plan de ordenación y manejo cuenca hidrográfica de los ríos Grande y Chico realizado por Corantioquia (2015) a escala 1:10.000 (gráfico 5c). La clasificación de la leyenda de la metodología Corine Land Cover (CLC) se usó para la clasificación y nomenclatura de las coberturas desarrollada por el IGAC en 2007. El mapa y estudio de suelos (escala 1: 50.000) realizado entre octubre de 2015 y marzo de 2016 se obtuvieron de la tesis doctoral realizada dentro del proyecto Utgra (2014) (gráfico 5d).

A partir de la información espacial, la cuenca se definió con un área de 103.434,77 hectáreas y se delineó en 20 subcuencas que representan los tributarios principales. Las clases de pendiente se definieron en cinco rangos (0-5 \%; 5-15\%; 15-40\%; 25-60\% y > 60 \%) y se delinearon 3.060 URH. La evapotranspiración real (AET) se calculó con base en la metodología desarrollada por Ritchie (1972), y la evapotranspiración potencial (PET) se simuló utilizando el método de Hargreaves y Samani (1985). Las prácticas actuales de manejo agrícola para estos sistemas de cultivos se simularon en el modelo como una línea de base. Los valores finales de los parámetros asociados con las operaciones de manejo se presentan en la tabla 6. Además, la base de 
datos de fertilizantes (.frt) se modificó de acuerdo con el fertilizante utilizado por los agricultores.

Gráfico 5. (a) Mapa de ubicación de estaciones meteorológicas, (b) modelo de elevación digital, (c) cobertura vegetal, (d) unidades de suelo

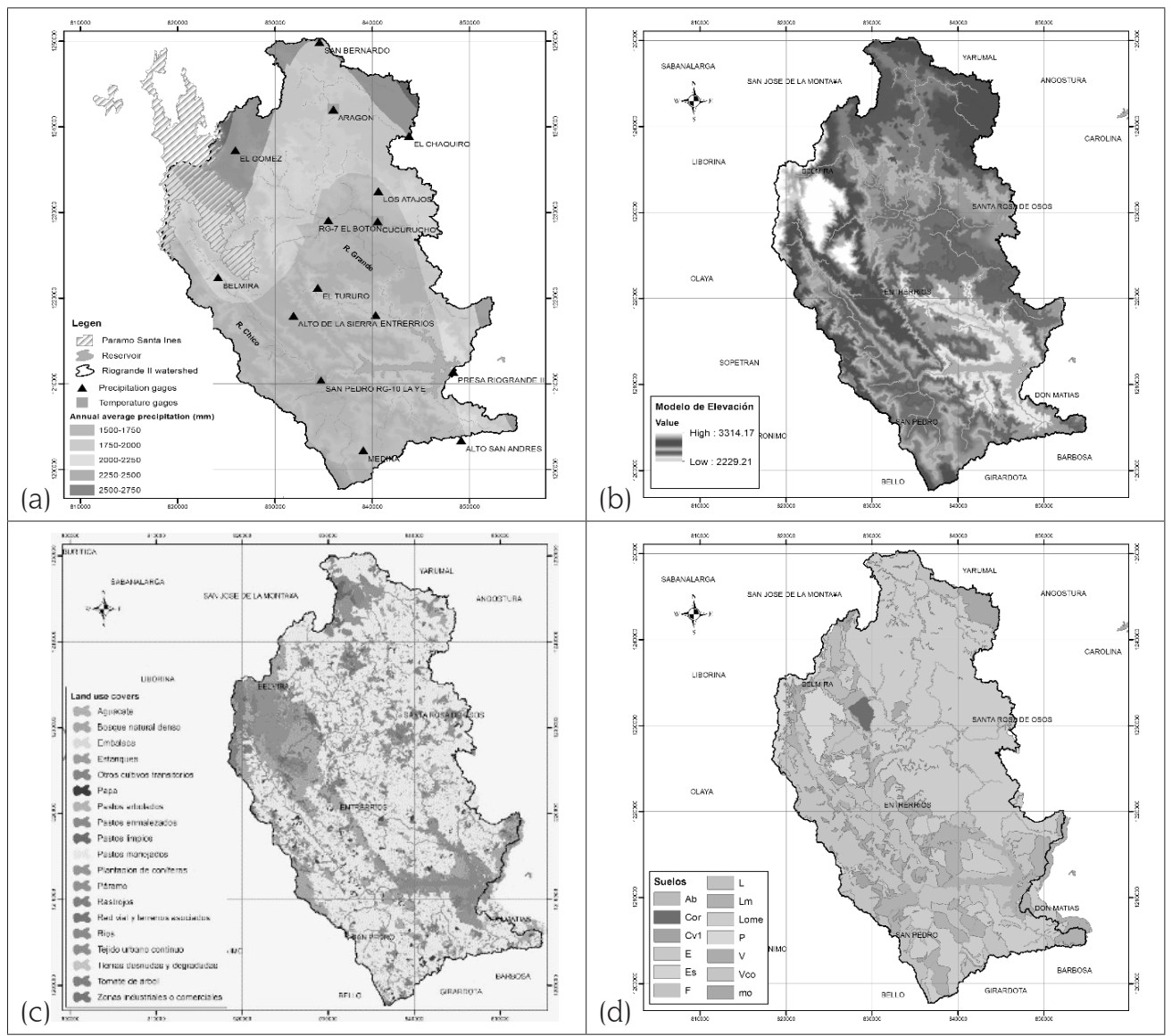

Fuente: Utgra (2018). 
Optimización de uso del suelo acorde con restricciones ambientales y sociales: caso cuenca de Riogrande II

Tabla 6. Valores de prácticas de manejo agrícola definidos en base de datos (.mgt file)

\begin{tabular}{|c|c|c|c|c|}
\hline \multirow[b]{2}{*}{ Nombre variable } & \multirow[b]{2}{*}{ Definición } & \multicolumn{3}{|c|}{ Valor definido } \\
\hline & & Papa & $\begin{array}{l}\text { Tomate } \\
\text { árbol }\end{array}$ & Kikuyo \\
\hline \multicolumn{5}{|c|}{ Siembra } \\
\hline Plant_ID & Código de la cobertura sembrada (.dat file) & POTA & TOMA & PASM \\
\hline Heat Units & $\begin{array}{l}\text { PHU: total de unidades térmicas requeri- } \\
\text { das por la planta para alcanzar punto de } \\
\text { madurez }\end{array}$ & 800 & 1.500 & 1.000 \\
\hline CN2 & $\begin{array}{l}\text { Initial SCS runoff curve number (Mín. } 35 \text { - } \\
\text { Máx. 98) }\end{array}$ & 62 & 60 & 62 \\
\hline \multicolumn{5}{|c|}{ Pastoreo } \\
\hline GRZ_DAYS & Número de días de pastoreo en cada HRU & & & 1 \\
\hline $\mathrm{BIO}_{-} \mathrm{EAT}$ & Materia seca consumida (kg/ha/día) & & & 51 \\
\hline $\mathrm{BIO} \_\mathrm{TRMP}$ & Materia seca pisoteada (kg/ha/día) & & & 650 \\
\hline Manure _KG & $\begin{array}{l}\text { Materia seca depositada como estiércol } \\
\text { (kg/ha/día) }\end{array}$ & & & 10 \\
\hline
\end{tabular}

\section{Labranza}

\begin{tabular}{ccccc}
\hline Tillage _ID & Maquinaria de labranza & Bedder & Bedder & Bedder \\
& Shaper & Disk & Disk \\
\hline
\end{tabular}

\section{Fertilización}

\begin{tabular}{llccc}
\hline FERT _ ID & Tipo de fertilizante/urea aplicada & 15-15-15 & $\begin{array}{c}\text { Urea+13 } \\
13-13\end{array}$ & $\begin{array}{c}\text { Urea }+ \\
\text { potreros }\end{array}$ \\
\hline FRT _ KG & $\begin{array}{l}\text { Cantidad de fertilizante/urea aplicada }(\mathrm{kg} / \\
\text { ha) }\end{array}$ & $\begin{array}{c}700 \\
(2 \text { por } \\
\text { ciclo) }\end{array}$ & $\begin{array}{c}530 \\
(7 \text { por } \\
\text { año) }\end{array}$ & $\begin{array}{c}90 \\
\text { (8 por año) }\end{array}$ \\
\hline FRT_Surface & $\begin{array}{l}\text { Fracción de fertilizantes aplicado } \\
\text { en los } 10 \mathrm{~mm}\end{array}$ & 0,2 & 0,9 & 0,9 \\
\hline
\end{tabular}

Fuente: Utgra (2018).

\subsubsection{Calibración y validación del modelo}

La calibración se realizó en varias etapas. Primero, se calcularon los coeficientes de Nash-Sutcliffe (NSE) y el error medio cuadrático (RMSE) para evaluar la eficiencia. En segundo lugar, para los cultivos de papa, tomate de árbol y kikuyo, se calibró el parámetro de rendimiento de cosecha (t/ha) comparando los valores promedio simulados con los valores de referencia. El modelo informa el rendimiento de la 
cosecha como peso seco, que representa una parte de la biomasa total producida diariamente después de la aplicación de cada operación de cosecha. Los valores de referencia empleados para el rendimiento promedio cosechado de la papa, tomate de árbol y kikuyo son de 8, 10 y 0,2 a 4 (t/ha), respectivamente. Los valores de referencia anuales definidos para comparar los rendimientos de la cosecha simulados durante el proceso de calibración fueron 16, 20 y 1,6 a 32 t/ha/año por cultivo, respectivamente. Finalmente, la calibración manual de las pérdidas de nutrientes se realizó entre 2014 y 2015, con datos discretos medidos para nitrato $-\mathrm{N}\left(\mathrm{NO}_{3}{ }^{-}-\mathrm{N} \mathrm{mg/l)} \mathrm{y} \mathrm{fósforo} \mathrm{soluble}\right.$ $\left(\mathrm{PO}_{4} \mathrm{mg} / \mathrm{l}\right)$ obtenidos de EPM. Estos datos incluyeron muestras tomadas para días específicos durante un estudio de campaña de monitoreo de la calidad del agua. Por lo tanto, los resultados se analizaron en promedios diarios y mensuales para las fechas correspondientes a los datos disponibles. Los valores promedio, mínimo y máximo se compararon entre los valores simulados y observados.

\subsection{Modelo económico}

El modelo económico estima el beneficio privado de las actividades agroindustriales dadas ciertas alternativas de rotación y las restricciones ambientales que garantizan la sostenibilidad. Se consideraron cuatro sistemas de rotación entre cultivos transitorios y permanentes. Estos sistemas se obtuvieron de las encuestas realizadas a los productores de las zonas al consultarles las prácticas económicas que tenían.

Tabla 7. Sistemas de rotación de cultivos

\begin{tabular}{|c|l|l|l|l|}
\hline Años simulados & Sistema de rotación 1 & Sistema de rotación 2 & Sistema de rotación 3 & Sistema de rotación 4 \\
\hline 2006 & Pasto kikuyo & Tomate de árbol & Tomate de árbol & Pasto kikuyo \\
\hline 2007 & Papa & Tomate de árbol & Tomate de árbol & Pasto kikuyo \\
\hline 2008 & Pasto kikuyo & Tomate de árbol & Tomate de árbol & Pasto kikuyo \\
\hline 2009 & Pasto kikuyo & Pasto kikuyo & Tomate de árbol & Pasto kikuyo \\
\hline 2010 & Papa & Pasto kikuyo & Tomate de árbol & Pasto kikuyo \\
\hline 2011 & Pasto kikuyo & Pasto kikuyo & Tomate de árbol & Pasto kikuyo \\
\hline 2012 & Pasto kikuyo & Pasto kikuyo & Tomate de árbol & Pasto kikuyo \\
\hline 2013 & Papa & Pasto kikuyo & Tomate de árbol & Pasto kikuyo \\
\hline 2014 & Pasto kikuyo & Pasto kikuyo & Tomate de árbol & Pasto kikuyo \\
\hline 2015 & Pasto kikuyo & Pasto kikuyo & Tomate de árbol & Pasto kikuyo \\
\hline
\end{tabular}

Fuente: Utgra (2018). 
Estas actividades productivas serán evaluadas bajo tres rangos de pisos térmicos en la cuenca (cuenca baja, media, alta), pues se reconoce que las dinámicas de uso del suelo son variables. Las limitantes ambientales estimadas con el modelo de simulación SWAT serán: tasas de erosión, escorrentía, generación de agua, nitratos y fosfatos generados por la aplicación de fertilizantes químicos y orgánicos.

Se formuló dentro del modelo otra restricción asociada a la extensión de suelo disponible para el uso de explotación agropecuaria en el territorio. Entre las limitantes o variables de restricción social o económica se incluye la mano de obra disponible y económicamente activa en el sector rural de los municipios de la cuenca. Las simulaciones se realizarán para un periodo máximo de diez años. La estructura general del modelo económico será maximizar el valor presente de los beneficios económicos del productor agropecuario dadas las condiciones ambientales de la cuenca, así:

$i=1,2,3$ tipos de cuenca.

$j=1, \ldots, 4$ sistemas de rotación de cultivos (C).

$\pi_{i j t}=$ beneficio neto $(\$ / \mathrm{ha})$ del sistema de rotación en los cultivos (C).

$x_{i j t}=$ hectáreas de uso del suelo para cultivos habituales o tradicionales $(\mathrm{C})$.

$c_{\mathrm{T}}=$ costo por t de concentrado comprada.

$\mathrm{T}_{i t}=\mathrm{t}$ de concentrado comprada.

$c_{\mathrm{V}, \mathrm{t}}=$ costo de compra de vacas por animal.

$\mathrm{V} c_{i t}=$ número de animales comprados.

$\mathrm{P}_{\mathrm{V}, \mathrm{it}}=$ precio de venta de vacas por animal.

$\mathrm{V} v_{i t}=$ número de animales vendidos.

$c_{\varepsilon, i t}=$ costo (\$/t) de extracción de sedimento.

$\varepsilon_{i t}=$ toneladas de extracción de sedimentos.

$\mathrm{M}_{i t}=$ toneladas de carne vendidas.

$\mathrm{P}_{\mathrm{M}, i t}=$ precio $(\$ / \mathrm{t})$ de venta de la tonelada de carne.

$\mathrm{K}_{i t}=$ miles de litros de leche vendidas.

$\mathrm{P}_{\mathrm{K}, i t}=$ precio $(\$ / 1000$ l) de venta de leche.

$\mathrm{CL}_{i t}=$ compra de jornales para uso en la finca.

$\mathrm{P}_{\mathrm{CL}, i t}=$ precio de compra de jornal para uso en la finca. 
$\mathrm{VL}_{i t}=$ venta de jornales para trabajos fuera de la finca.

$\mathrm{P}_{\mathrm{VL}, i t}=$ precio de venta de jornal para trabajos fuera de la finca.

$\tau_{t}=$ transferencias netas.

$\mu=$ deuda total durante los $t$ periodos.

$\mathrm{R}_{i t}=$ miles de litros de aguas residuales vertidas en la cuenca.

El modelo económico de optimización del uso del suelo del productor agropecuario está dado por:

$\pi_{i j t \mathrm{C}}, \pi_{i k t \mathrm{~B}}, \pi_{i l t \mathrm{~F}}, \mathrm{~T}_{i t}^{\max }, \mathrm{Vc}_{i t}, \mathrm{Vv}_{i t}, \varepsilon_{i t}, \mathrm{MP}_{i t}{ }^{\prime} \mathrm{VPN}(\mathrm{BN})=\begin{aligned} & \text { valor presente neto de los beneficios } \\ & \text { del productor agropecuario }\end{aligned}$
$\mathrm{M}_{i t}, \mathrm{~K}_{i t}, \mathrm{MN}_{i t}, \mathrm{~W}_{i t}, \mathrm{CO}_{i t}, \mathrm{CL}_{i t}, \mathrm{VL}_{i t}$

$Z=$ Ingresos por uso agrícola del suelo+transferencias-deuda+ingresos netos por actividades económicas

$$
\begin{aligned}
= & \sum_{i=1}^{3} \sum_{j=1}^{12} \sum_{t=0}^{9} \frac{\pi_{i j t \mathrm{C}} x_{i j t \mathrm{C}}}{(1+r)^{-t}}+\sum_{t=0}^{9} \frac{\tau_{t}}{(1+r)^{-t}}-\mu \\
& +\sum_{t=1}^{3} \sum_{t=0}^{9} \frac{-c_{\mathrm{T}, i t} \mathrm{~T}_{i t}-c_{\mathrm{V}, i t} \mathrm{~V}_{i t}+\mathrm{P}_{\mathrm{V}, i t} \mathrm{~V}_{i t}-c_{\varepsilon, i t} \varepsilon_{i t}+\mathrm{P}_{\mathrm{M}, i t} \mathrm{M}_{i t}+\mathrm{P}_{\mathrm{K}, i t} \mathrm{~K}_{i t}-\mathrm{P}_{\mathrm{CL}, i t} \mathrm{CL}_{i t}+\mathrm{P}_{\mathrm{VL}, i t} \mathrm{VL}_{i t}}{(1+r)^{-t}}
\end{aligned}
$$

Sujeto a:

Uso de suelo: $\sum_{j=1}^{4} x_{i j \mathrm{c}} \leq \mathrm{H}_{i} \quad i=1,2,3$ donde $\sum_{i=1}^{3} \mathrm{H}_{i}=\mathrm{H}$

Niveles de erosión: $\sum_{i=1}^{3} \sum_{j=1}^{4} s_{i t c} x_{i j t c} \leq S_{t}$

Niveles de agua generada: $-\sum_{i=1}^{3} \sum_{j=1}^{4} q_{i t c} \chi_{i j t c}-\sum_{i=1}^{3} q_{i t V} V_{i t} \leq 0$

Niveles de nitratos: $\sum_{i=1}^{3} \sum_{j=1}^{4} n_{i t \mathrm{C}} \chi_{i j t \mathrm{C}}+\sum_{i=1}^{3}\left(n_{i t \mathrm{~T}} \mathrm{~T}_{i t}-n_{i t \varepsilon} \varepsilon_{i t}+n_{i t \mathrm{R}}\right) \leq \mathrm{N}_{t}$

Niveles de fosfatos: $\sum_{i=1}^{3} \sum_{j=1}^{4} f_{i t \mathrm{C}} x_{i j t \mathrm{C}}+\sum_{i=1}^{3}\left(f_{i t \mathrm{~T}} \mathrm{~T}_{i t}-f_{i t \varepsilon} \varepsilon_{i t}+f_{i t \mathrm{R}}\right) \leq \mathrm{F}_{t}$

Niveles de secuestración $\mathrm{CO}_{2}:-\sum_{i=1}^{3} \sum_{j=1}^{12} \mathrm{CO}_{i t \mathrm{C}} \chi_{i j t \mathrm{C}} \leq 0$

Mano de obra disponible: $\sum_{j=1}^{4} \mathrm{~L}_{i j \mathrm{c}} \chi_{i j t \mathrm{c}}+\sum_{i=1}^{3}\left(\mathrm{CL}_{i t}-\mathrm{VL}_{i t}\right) \leq \mathrm{L}_{i} \quad$ donde $\sum_{i=1}^{3} \mathrm{~L}_{i}=\mathrm{L}$ 
Energía (e) para ganado producida: $-\sum_{i=1}^{3} \sum_{j=1}^{4} e_{i i t \mathrm{c}} x_{i j \mathrm{tc}}-\sum_{l=1}^{3}\left(e_{i t \mathrm{t}} \mathrm{T}_{i t}+e_{i t \mathrm{~V}} \mathrm{~V} c_{i t}-e_{i t \mathrm{~V}} \mathrm{~V} v_{i t}\right) \leq 0$

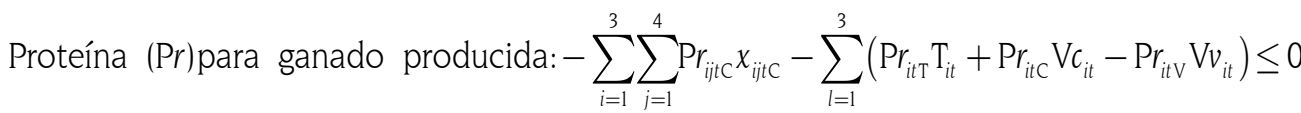

Número de vacas en la cuenca:

$$
\sum_{i=1}^{3}\left(\mathrm{~V} c_{i t}-\mathrm{V} v_{i t}\right)=0
$$

Miles de litros de leche producida:

$$
\sum_{i=1}^{3}\left(\mathrm{~K}_{\text {itProducida }}-\mathrm{K}_{i t}\right)=0
$$

Toneladas de carne producida:

$$
\sum_{i=1}^{3}\left(\mathrm{M}_{i \text { tProducida }}-\mathrm{M}_{i t}\right)=0
$$

$x_{i j t} \geq 0 \quad t=1, \ldots, 10 ; \quad j=1, \ldots, 12 ; \quad i=1,2,3 ; \mathrm{C}=$ cultivos tradicionales; $\mathrm{B}=$ cultivos silvícolas; $\mathrm{F}=$ cultivos forrajeros.

Fuente: adaptado de Quintero, Estrada y García (2006).

Teniendo que:

$\mathrm{H}_{i}=$ número total de hectáreas.

$s_{i j t}=$ estimación de niveles de erosión anuales en toneladas por hectárea al año.

$\mathrm{S}_{t}=$ máximo aceptable en toneladas por hectárea de erosión.

$q_{i j t}=$ estimación de agua generada en metros cúbicos por hectárea.

$n_{i j t}=$ niveles de nitratos en toneladas por hectárea.

$f_{i j t}=$ niveles de fosfatos en toneladas por hectárea.

$\mathrm{N}_{t}$ y $\mathrm{F}_{t}=$ niveles máximos aceptables en toneladas por hectárea de nitratos y fosfatos respectivamente.

$\mathrm{CO}_{i j t}=$ niveles de secuestro de $\mathrm{CO}_{2}$ en toneladas por hectárea.

$\mathrm{L}_{i j t}=$ mano de obra disponible.

$\mathrm{L}_{t}=$ mano de obra rural económicamente activa en los municipios de la cuenca.

$e_{i j t}$ y $\mathrm{Pr}_{i j t}=$ niveles de energía y proteína para ganado generadas en toneladas por hectárea

$V_{i j t}=$ número de vacas existentes en la finca a partir del balance entre compra y venta de animales.

$\mathrm{K}_{i j \mathrm{t}}=$ toneladas de leche producidas y vendidas.

$\mathrm{M}_{i j t}=$ toneladas de carne producidas y vendidas. 
Al modelo se ingresó la información sobre sistemas de producción agropecuaria, producción ganadera y externalidades.

\section{RESULTADOS}

\subsection{Modelo hidrológico}

\subsubsection{Caudal y sedimentos}

Los resultados de calibración y validación del caudal mensual se muestran en los gráficos 6a, 6b y 6c. Los valores NSE obtenidos para las tres estaciones de aforo fueron 0,88 ; 0,85 y 0,93 para el período de calibración y 0,86; 0,85 y 0,77 para el período de validación. De acuerdo con las pautas de calificación de desempeño desarrolladas por Moriasi et al. (2007), la calibración y la validación mensuales del flujo de la corriente se consideran muy buenas.

Gráfico 6. Promedio mensual caudal simulado vs. aforado en (a) Puente Belmira, (b) San Pedro RG-10 La Ye y (c) Presa
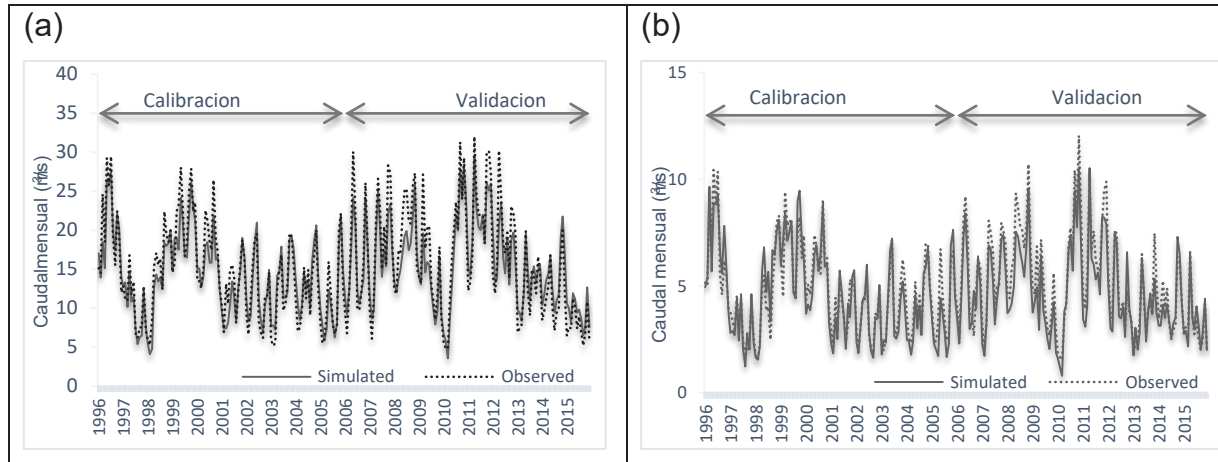

(c)

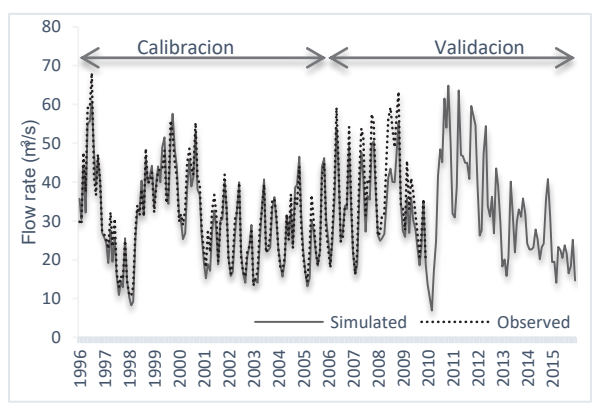

Fuente: elaboración propia.

El modelo tiene como salida para cada una de las URH los valores obtenidos en cada uno de los componentes del balance hídrico: precipitación anual (mm), 
evapotranspiración real $(\mathrm{mm})$, percolación $(\mathrm{mm})$, escorrentía $(\mathrm{mm})$, pérdida por transmisión (mm), flujo lateral (mm), agua aportada por el acuífero superficial ( $\mathrm{mm}$ ) y producción de agua $(\mathrm{H} 2 \mathrm{O} \mathrm{mm}$ ): monto total de agua que sale de cada URH y de entrar al canal principal durante el paso del tiempo (WYLD = SURQ + LATQ + GWO - Tloss - abstracciones estanque). Con esta información se procede a graficar la contribución de agua al caudal por parte de cada UHR (gráfico 7). La contribución de agua al caudal es el promedio multianual (1995-2015) de la producción de agua (mm) por cada URH. Dentro de las áreas prioritarias por el aporte de agua al caudal en la cuenca de Riogrande II, se identificaron que las coberturas asociadas a la mayor producción de agua corresponden a los bosques plantados (PINA), bosque natural fragmentado (MESO), mosaico de cultivos pastos y espacios naturales (RYEG), arbustos matorrales y pastos enmalezados (RYEE), pastos manejados (PASM), tejido urbano (URHD) entre otras.

Gráfico 7. Producción de agua anual por cada URH

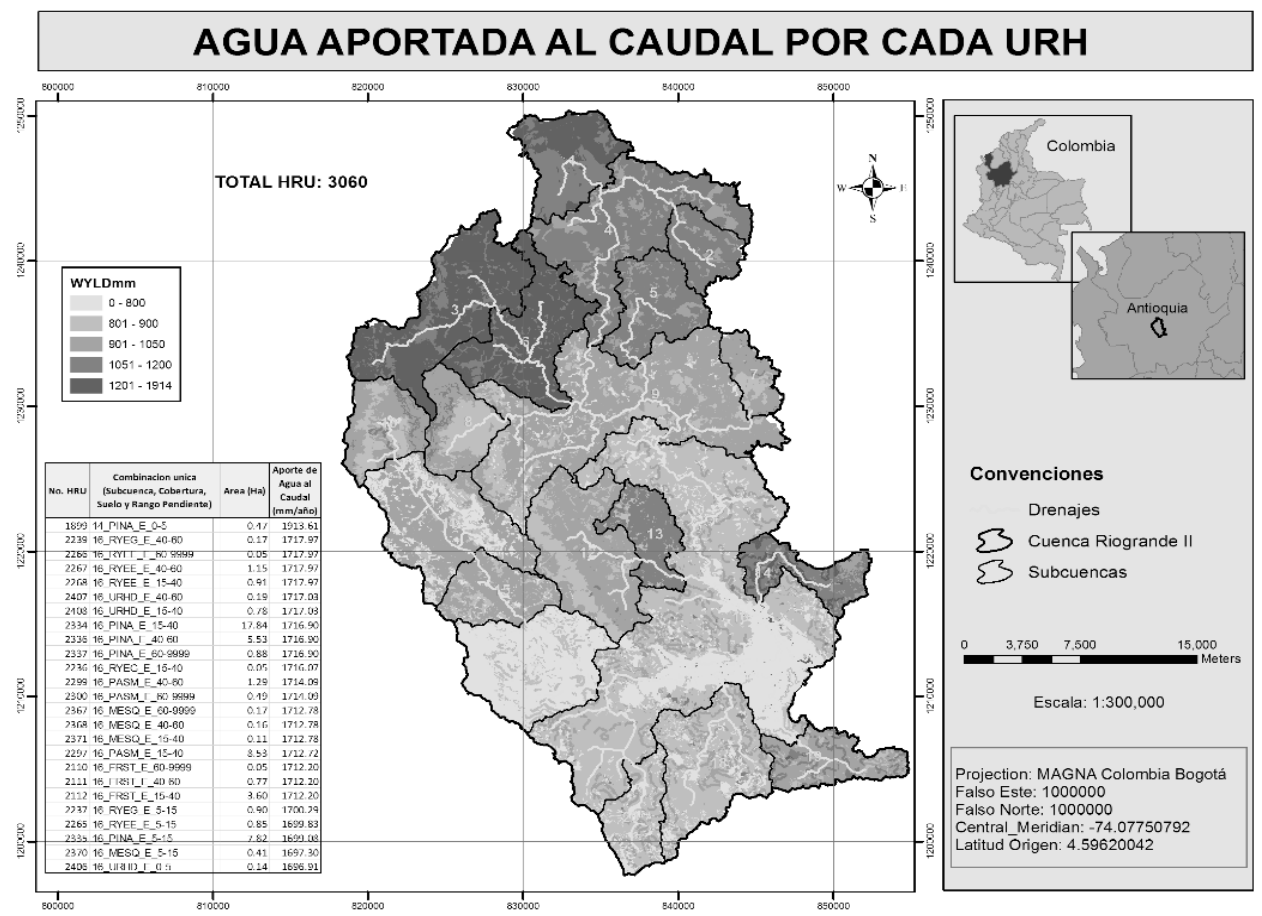

Fuente: elaboración propia.

Existe muy poca información de sedimentos para realizar una calibración y validación de los resultados obtenidos en la modelación, sin embargo, se realizó una comparación del promedio anual simulado con un valor estimado en base 
a los registros de batimetrías realizadas en el embalse entre 1996-2009. El valor definido del promedio anual de sedimentos registrado para la cuenca Riogrande es de 7,32 (t/ha/año) y el valor simulado obtenido es de 12,4 (t/ha/año). Se aprecia que el promedio simulado de sedimentos anual por hectárea es mucho menor al promedio registrado en las batimetrías. Sin una calibración periódica es muy difícil estimar un valor que corresponda a la realidad de la cuenca. Adicionalmente, el modelo no simula procesos de remoción en masa que pudiesen haber ocurrido en este periodo. Los resultados de sedimentos presentados no se pueden soportar de forma cuantitativa, pero sí se puede definir cuáles son las áreas (URH), coberturas y suelos que presentan mayor y menor producción de sedimentos en forma cualitativa.

\subsubsection{Productividad de cultivos}

La calibración del rendimiento de los cultivos se realizó mensualmente, específicamente en los meses en que se implementó la operación de cosecha en cada uno de los programas de manejo de papa, tomate de árbol y kikuyo. Los rendimientos obtenidos por ciclo y anuales de cada cultivo se presentan en la tabla 8. Los rendimientos anuales cosechados de papa a nivel de HRU están entre 10 y 26 t/ha/año, con un rendimiento promedio anual de 16 t/ha/año. Para tomate de árbol es de 20 t/ha/año, con valores que varían entre 8 y 34 t/ha/año. El pasto kikuyo está presente en toda la cuenca, excepto en la subcuenca 8. El área plantada corresponde al 58,31 $\%$ del área total de la cuenca. Los valores de rendimiento cosechados varían entre 0,8 y 72 t/ha/año, con un rendimiento promedio anual de 16 t/ha/año. En general, las predicciones del rendimiento del cultivo adquiridas por la calibración manual fueron satisfactorias.

Tabla 8. Productividad simulada por cultivo

\begin{tabular}{ccccccc}
\hline Valores & \multicolumn{3}{c}{$\begin{array}{c}\text { Productividad por ciclo } \\
(t / h a / c i c l o)\end{array}$} & \multicolumn{4}{c}{$\begin{array}{c}\text { Productividad por año } \\
(t / \text { ha/ciclo })\end{array}$} \\
\hline Papa & $\begin{array}{c}\text { Tomate } \\
\text { árbol }\end{array}$ & $\begin{array}{c}\text { Pasto } \\
\text { kikuyo }\end{array}$ & $\begin{array}{c}\text { Papa } \\
(2 \text { cosechas })\end{array}$ & $\begin{array}{c}\text { Tomate árbol } \\
(2 \text { cosechas })\end{array}$ & $\begin{array}{c}\text { Pasto kikuyo } \\
(8 \text { pastoreos })\end{array}$ \\
\hline Mín. & 5 & 4 & 0,1 & 10 & 8 & 0,8 \\
\hline Máx. & 13 & 17 & 9 & 26 & 34 & 72 \\
\hline Promedio & 8 & 10 & 2 & 16 & 20 & 16 \\
\hline
\end{tabular}

Fuente: elaboración propia.

\subsubsection{Nutrientes en escorrentía}

Se contó con información de parámetros de calidad de agua monitoreada con muestreos para los años del 2014 y 2015 en la entrada del río Chico al embalse 
( $\mathrm{N} \mathrm{06}^{\circ} 30.390^{\prime} \mathrm{W} 075^{\circ} 32.059^{\prime}$ ). El gráfico 8 presenta los resultados obtenidos de la validación de los resultados de nitrato $\left(\mathrm{NO}_{3}-\mathrm{N}\right)$ y fósforo soluble mensuales para el periodo disponible de aforos realizados. Para $\mathrm{NO}_{3}-\mathrm{N}$ y $\mathrm{PO}_{4}$, los valores mensuales de NSE fueron 0,48 y 0,33, respectivamente, lo que indica que los resultados no son satisfactorios en términos de valores extremos, siguiendo a Moriasi et al. (2007). Sin embargo, las tendencias se ajustaron adecuadamente con valores de $\mathrm{R}^{2}$ de 0,81 y 0,59 , respectivamente. Los rendimientos de la simulación de nutrientes están directamente relacionados con la simulación de escorrentía (ecuación 2), por lo tanto, las incertidumbres en las estimaciones de escorrentía se transfieren a la predicción de la carga de nutrientes.

En general, la inspección visual de los resultados encuentra una coincidencia temporal relativamente buena entre los valores simulados y observados. Las mayores incertidumbres ocurren en el evento extremo en marzo de 2014 y agosto de 2015 para los componentes de nitrógeno, y en abril y mayo de 2014 para el fósforo mineral (gráfico 8). Existe un vínculo directo entre los rendimientos de nutrientes y la estacionalidad de la precipitación y escorrentía en la cuenca de Riogrande. Sin embargo, vale la pena mencionar que se dio prioridad a la representación de las cargas de nutrientes simuladas con la información disponible.

\section{Gráfico 8. Valores mensuales de $\mathrm{NO}_{3}-\mathrm{N}$ (izquierda) y soluble $\mathrm{P}$ (derecha) simulados y aforados}
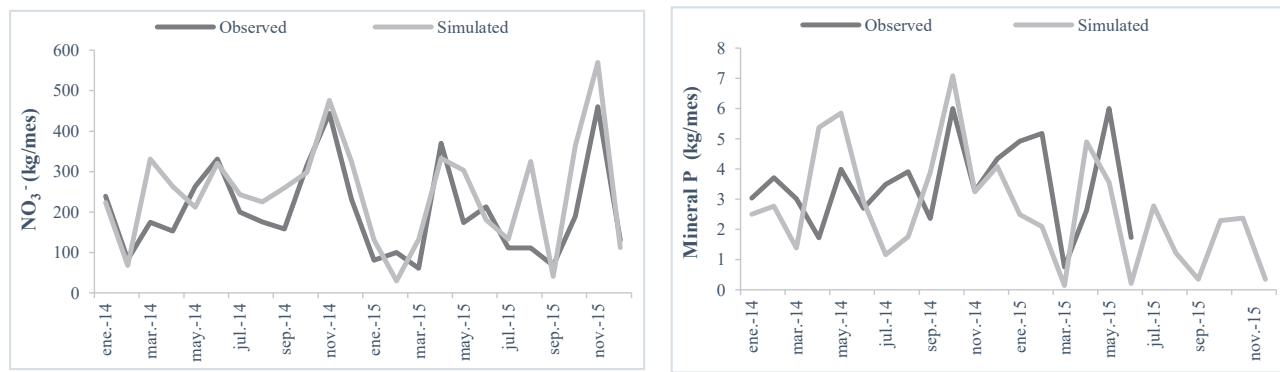

Fuente: elaboración propia.

Se seleccionaron algunos años en la simulación del período total por tener la mayor precipitación promedio anual, para representar gráficamente la variabilidad espacial mensual del $\mathrm{NO}_{3}-\mathrm{N}$ y el fósforo soluble. La mayor recurrencia de aporte de nutrientes al caudal por mes ocurrió en las subcuencas 4, 5, 7 y 9 (parte alta de la cuenca) y 12, 15, 17, 19 y 20 (parte baja de la cuenca) para $\mathrm{NO}_{3}{ }^{-}-\mathrm{N}$ y P soluble (gráficos 9 y 10). En las subcuencas identificadas con el mayor aporte de nutrientes están presentes los tres cultivos estudiados. Las plantaciones del cultivo de papa y tomate de árbol tienen niveles medios de aporte, los pastos manejados aportan el mayor nitrato $\left(\mathrm{NO}_{3}\right)$ y fósforo soluble transportados por escorrentía. 
Los resultados mostraron una fuerte asociación entre la estacionalidad de la precipitación, los programas de manejo agrícola (sistema de cultivos y pastos) y la dinámica espacial del aporte de nutrientes en escorrentía. Sin embargo, el mayor aporte de nutrientes al caudal se produce en la región con la precipitación más baja en la cuenca de Riogrande, lo que hace necesario mejorar las prácticas del calendario de fertilización para evitar coincidir con los meses de lluvia.

Gráfico 9. Comportamiento espacial mensual de nitratos
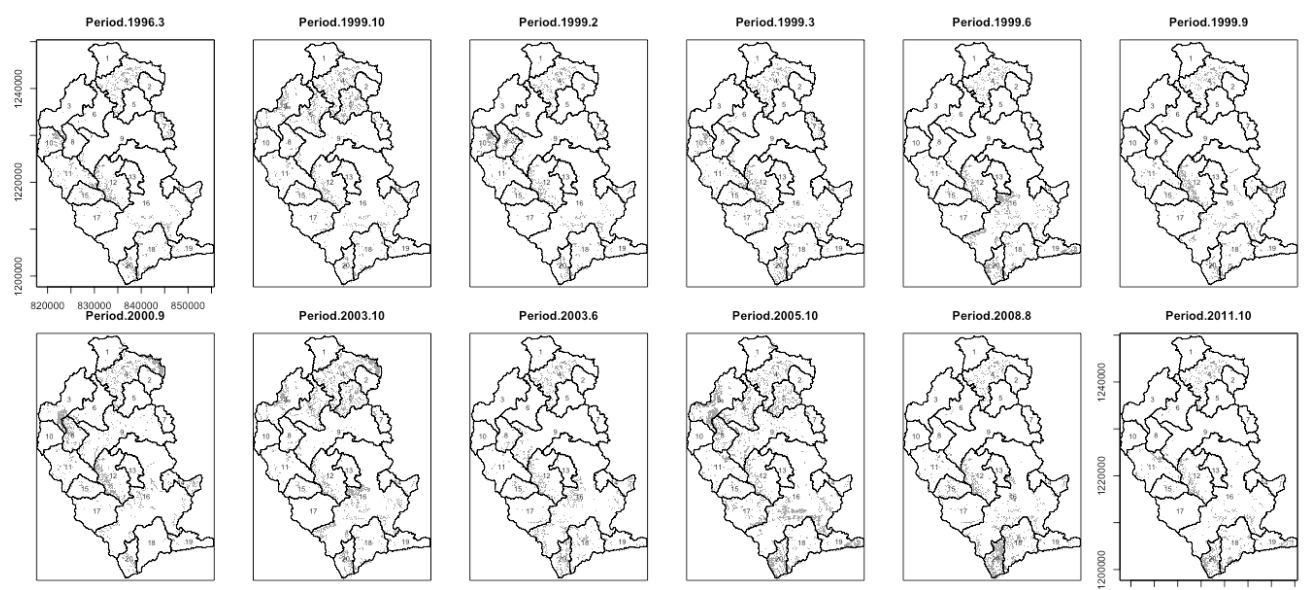

Fuente: elaboración propia

Gráfico 10. Comportamiento espacial mensual del fosforo soluble
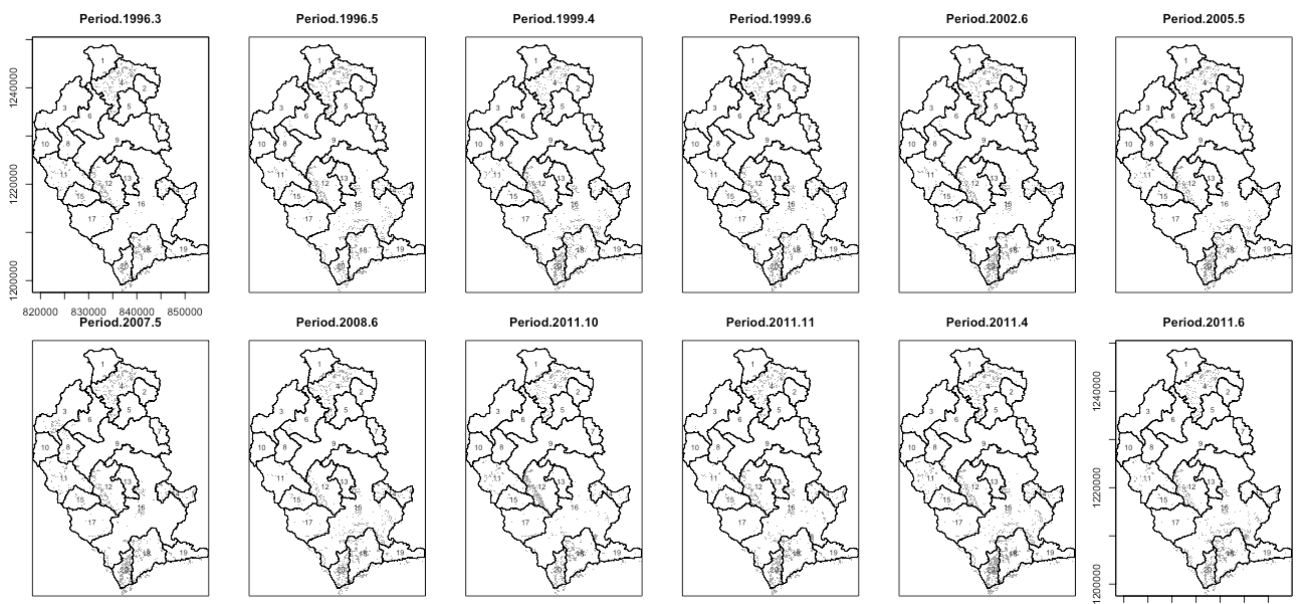

Fuente: elaboración propia 


\subsection{Modelo económico}

Los resultados principales indican la asignación óptima de hectáreas por URH y el sistema de rotación que asegura el cumplimiento de las restricciones. De los sistemas de rotación actualmente utilizados el que combina pastos con cultivos de papa (sistema 1) es el único que logra cumplir con las restricciones impuestas al modelo y que, por tanto, asegura la sostenibilidad a largo plazo de la cuenca. Los resultados presentados en la tabla 9 dan cuenta del número máximo de hectáreas que debe dedicarse a este sistema de rotación para cada una de las subcuencas del sistema.

Tabla 9. Resumen de resultados del uso de suelo

\begin{tabular}{|c|c|c|c|c|c|c|c|}
\hline \multicolumn{8}{|c|}{ Asignación óptima en hectáreas de uso del suelo por sistemas de rotación } \\
\hline \multirow{2}{*}{ Cuenca } & \multirow{2}{*}{ Subcuenca } & \multirow{2}{*}{ Uso actual } & \multicolumn{4}{|c|}{ Sistemas de rotación } & \multirow{2}{*}{ Reducción } \\
\hline & & & 1 & 2 & 3 & 4 & \\
\hline \multirow{15}{*}{ Cuenca alta } & 1 & 1.511 & 1.511 & 0 & 0 & 0 & $0 \%$ \\
\hline & 2 & 1.633 & 1.633 & 0 & 0 & 0 & $0 \%$ \\
\hline & 3 & 2.827 & 2.285 & 0 & 0 & 0 & $19 \%$ \\
\hline & 4 & 5.017 & 2.285 & 0 & 0 & 0 & $54 \%$ \\
\hline & 5 & 2.705 & 2.285 & 0 & 0 & 0 & $16 \%$ \\
\hline & 6 & 3.088 & 2.285 & 0 & 0 & 0 & $26 \%$ \\
\hline & 7 & 920 & 920 & 0 & 0 & 0 & $0 \%$ \\
\hline & 8 & 0 & 0 & 0 & 0 & 0 & $0 \%$ \\
\hline & 9 & 7.673 & 2.285 & 0 & 0 & 0 & $70 \%$ \\
\hline & 10 & 242 & 242 & 0 & 0 & 0 & $0 \%$ \\
\hline & 11 & 2.958 & 2.285 & 0 & 0 & 0 & $23 \%$ \\
\hline & 12 & 3.567 & 2.285 & 0 & 0 & 0 & $36 \%$ \\
\hline & 13 & 1.901 & 1.901 & 0 & 0 & 0 & $0 \%$ \\
\hline & 15 & 3.610 & 2.285 & 0 & 0 & 0 & $37 \%$ \\
\hline & 17 & 13.063 & 2.285 & 0 & 0 & 0 & $83 \%$ \\
\hline \multirow{2}{*}{$\begin{array}{c}\text { Cuenca } \\
\text { media }\end{array}$} & 14 & 1.705 & 1.705 & 0 & 0 & 0 & $0 \%$ \\
\hline & 16 & 1.454 & 1.454 & 0 & 0 & 0 & $0 \%$ \\
\hline \multirow{3}{*}{ Cuenca baja } & 18 & 3.954 & 2.285 & 0 & 0 & 0 & $42 \%$ \\
\hline & 19 & 2.001 & 2.001 & 0 & 0 & 0 & $0 \%$ \\
\hline & 20 & 1.718 & 1.718 & 0 & 0 & 0 & $0 \%$ \\
\hline
\end{tabular}


Juan David Osorio Múnera, Natalia Uribe Rivera, Lina Marcela García-Tavera y Eduardo Andrés Bustamante Ochoa

\begin{tabular}{|c|c|c|c|c|c|c|c|}
\hline \multicolumn{8}{|c|}{ Asignación óptima en hectáreas de uso del suelo por sistemas de rotación } \\
\hline \multirow{2}{*}{ Cuenca } & \multirow{2}{*}{ Subcuenca } & \multirow{2}{*}{ Uso actual } & \multicolumn{4}{|c|}{ Sistemas de rotación } & \multirow{2}{*}{ Reducción } \\
\hline & & & 1 & 2 & 3 & 4 & \\
\hline \multirow[t]{4}{*}{ Cuenca alta } & 1 & 1.511 & 1.511 & 0 & 0 & 0 & $0 \%$ \\
\hline & 2 & 1.633 & 1.633 & 0 & 0 & 0 & $0 \%$ \\
\hline & 3 & 2.827 & 2.285 & 0 & 0 & 0 & $19 \%$ \\
\hline & 4 & 5.017 & 2.285 & 0 & 0 & 0 & $54 \%$ \\
\hline \multicolumn{8}{|c|}{ Asignación óptima en hectáreas de uso del suelo por sistemas de rotación } \\
\hline \multirow{12}{*}{ Cuenca } & Cubsune & & \multicolumn{4}{|c|}{ Sistemas de rotación } & \multirow{2}{*}{ Reducción } \\
\hline & suocuenca & Uso actual & 1 & 2 & 3 & 4 & \\
\hline & 6 & 3.088 & 2.285 & 0 & 0 & 0 & $26 \%$ \\
\hline & 7 & 920 & 920 & 0 & 0 & 0 & $0 \%$ \\
\hline & 8 & 0 & 0 & 0 & 0 & 0 & $0 \%$ \\
\hline & 9 & 7.673 & 2.285 & 0 & 0 & 0 & $70 \%$ \\
\hline & 10 & 242 & 242 & 0 & 0 & 0 & $0 \%$ \\
\hline & 11 & 2.958 & 2.285 & 0 & 0 & 0 & $23 \%$ \\
\hline & 12 & 3.567 & 2.285 & 0 & 0 & 0 & $36 \%$ \\
\hline & 13 & 1.901 & 1.901 & 0 & 0 & 0 & $0 \%$ \\
\hline & 15 & 3.610 & 2.285 & 0 & 0 & 0 & $37 \%$ \\
\hline & 17 & 13.063 & 2.285 & 0 & 0 & 0 & $83 \%$ \\
\hline \multirow{2}{*}{$\begin{array}{c}\text { Cuenca } \\
\text { media }\end{array}$} & 14 & 1.705 & 1.705 & 0 & 0 & 0 & $0 \%$ \\
\hline & 16 & 1.454 & 1.454 & 0 & 0 & 0 & $0 \%$ \\
\hline \multirow{3}{*}{ Cuenca baja } & 18 & 3.954 & 2.285 & 0 & 0 & 0 & $42 \%$ \\
\hline & 19 & 2.001 & 2.001 & 0 & 0 & 0 & $0 \%$ \\
\hline & 20 & 1.718 & 1.718 & 0 & 0 & 0 & $0 \%$ \\
\hline
\end{tabular}

Fuente: elaboración propia.

Comparando los resultados obtenidos de la optimización con el uso actual podemos observar que es necesaria una reducción aproximada del $42 \%$ del número de hectáreas dedicadas a la producción. A partir de los resultados de la tabla 10, se tiene que para asegurar la sostenibilidad de la cuenca la reducción debe ser principalmente en las zonas de cuenca alta y baja . 
Optimización de uso del suelo acorde con restricciones ambientales y sociales: caso cuenca de Riogrande II

Tabla 10. Hectáreas usadas para actividades productivas de siembra de pastos, papa y tomate de árbol

\begin{tabular}{lccc}
\hline \multicolumn{1}{c}{ Ubicación } & Uso actual en hectáreas & $\begin{array}{c}\text { Modelación línea base en } \\
\text { hectáreas }\end{array}$ & $\begin{array}{c}\text { Porcentaje de cambio en el } \\
\text { uso del suelo }\end{array}$ \\
\hline Cuenca alta & 50.715 & 26.775 & $47 \%$ \\
\hline Cuenca media & 3.158 & 3.158 & $0 \%$ \\
\hline Cuenca baja & 7.673 & 6.004 & $22 \%$ \\
\hline Total & 61.546 & 35.937 & $42 \%$ \\
\hline
\end{tabular}

Fuente: elaboración propia.

Los resultados en términos ambientales están condicionados por las metas propuestas en las restricciones. Para los periodos analizados los niveles máximos de erosión, nitratos y fosfatos que aseguren la sostenibilidad de la cuenca se presentan en la tabla 11.

Tabla 11. Valores máximos de erosión, nitratos y fosfatos.

\begin{tabular}{|l|c|c|c|}
\hline \multicolumn{4}{|c|}{ Valores máximos variables ambientales } \\
\hline \multicolumn{1}{|c|}{ Cuenca } & Erosión $(\mathrm{T} / \mathrm{ha})$ & Nitratos $(\mathrm{kg} / \mathrm{ha})$ & Fosfatos $(\mathrm{kg} / \mathrm{ha})$ \\
\hline Cuenca alta & 136.641 & 206.796 & 157.759 \\
\hline Cuenca media & 34.884 & 53.647 & 44.750 \\
\hline Cuenca baja & 46.166 & 73.641 & 61.165 \\
\hline Total cuenca & 217.692 & 334.085 & 263.675 \\
\hline
\end{tabular}

Fuente: elaboración propia.

El gráfico 11 muestra los resultados totales por año de la optimización para cada una de las variables ambientales. Hay algunos picos de comportamiento para estas que corresponden a los años 2, 5 y 8. Acorde con el sistema escogido como óptimo durante estos años se implementan cultivos transitorios de papa como precursores de papa. Esto muestra que hay un peor desempeño en términos ambientales de los cultivos transitorios respecto de los pastos, lo que corresponde con los resultados hallados en el modelo hidrológico. Sin embargo, la elección de este sistema de rotación tiene sentido en la medida en la que el modelo tiene en cuenta también variables de índole económico. Así, las desmejoras ambientales durante los periodos de siembra de papa se compensan con la rotación con otros cultivos, lo que permite mejorar la productividad y los beneficios económicos asociados. 
Gráfico 11. Resultados de a. erosión, b. nitratos y c. fosfatos (total por año)

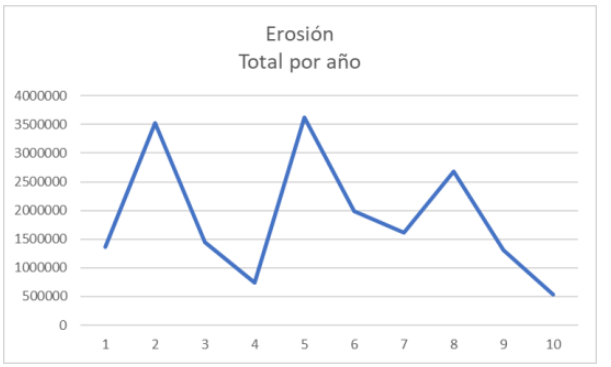

a

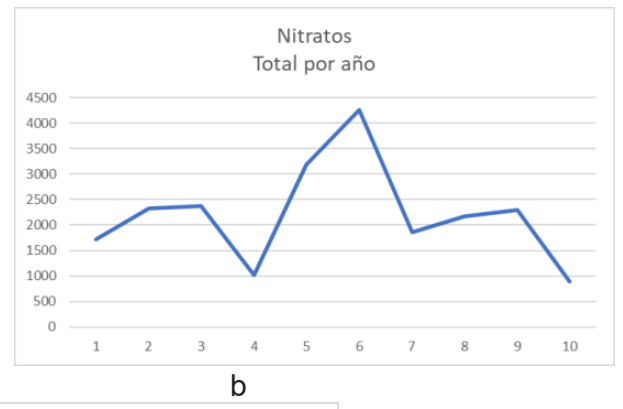

b

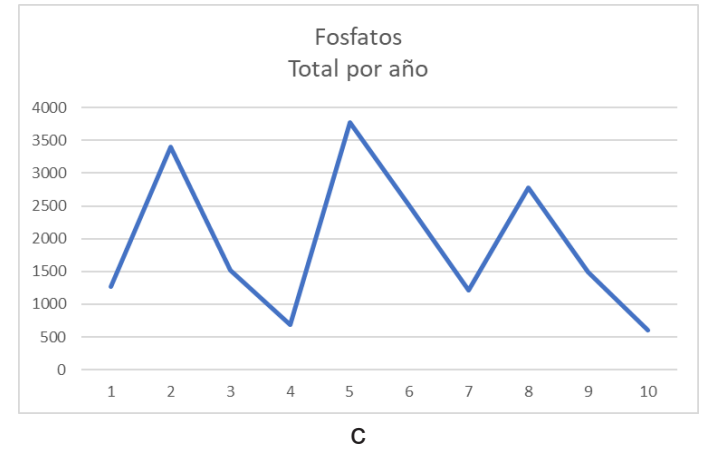

Fuente: elaboración propia.

\section{CONCLUSIONES}

El ejercicio es una mirada integral que consideró la importancia del recurso hídrico en las dinámicas económicas y ambientales de la cuenca de Riogrande II y los diferentes usos del suelo que actualmente se realizan en el territorio donde las poblaciones rurales dependen de la variedad de recursos producidos en la cuenca y que con las técnicas tradicionales de cultivo generan presión y degradación del suelo y contaminación del recurso hídrico a través de la deforestación, la ganadería extensiva y el uso excesivo de fertilizantes. El modelo de optimización aplicado es una primera aproximación a la gestión de los recursos naturales en el territorio, permite identificar los valores óptimos de las variables de decisión de uso del suelo que maximicen la función objetivo de valor presente de los beneficios netos de la población cumpliendo con restricciones impuestas sobre variables de interés ambiental.

Para lograr unos niveles de sostenibilidad de la cuenca acordes con los límites establecidos en las restricciones, es necesario que se implemente el sistema de rotación 1, correspondiente a una combinación de pastos y papa, ya que este sistema es el que genera el mayor ingreso posible acorde con las restricciones. Se requiere también una reducción de alrededor del 42 \% del área total dedicada a 
las actividades productivas, especialmente en el área correspondiente a la cuenca alta y cuenca baja.

Los resultados arrojados por el modelo hidrológico permiten afirmar que, en general, el pastoreo rotacional permite una mayor capacidad de carga, mejor aprovechamiento de fertilizantes, facilidad en el manejo de los animales, control de especies no deseables, crecimiento más uniforme de la pastura, menor incidencia parasitaria, entre otras ventajas. Es necesario confrontar algunas limitantes del sistema como: una mayor compactación del suelo por incremento de su uso que deriva en niveles incontrolables de erosión, mayores requerimientos de mano de obra y labores administrativas, mayor inversión en cercos, bebederos, saladeros. Los cultivos de papa y pastos aportan los mayores niveles de nitrógeno y fósforo orgánico en la cuenca. Los cultivos transitorios aportan el mayor nitrato (NO3) transportado en la escorrentía, con el cultivo de papa en segundo lugar.

\section{BIBLIOGRAFÍA}

Arnold, Jeff; Srinivasan, Raghunatan; Muttiah, Ranjan y Williams, Jimmy (1998). Large area hydrologic modeling and assessment. Part I: Model development. En: JAWRA Journal of the American Water Resources Association, vol. 34, n. ${ }^{\circ}$ 1, p. 73-89.

Cárdenas, María Fernanda (2013). La gestión de ecosistemas estratégicos proveedores de agua. En: Gestión y Ambiente, vol. 16, n.․ 1, p. 109-122.

Corantioquia - Corporación Autónoma Regional del Centro de Antioquia. (2015). Actualización y ajuste Plan de Ordenación y Manejo de la Cuenca de los Ríos Grande y Chico. Medellín: Corantioquia, $768 \mathrm{p}$.

Corantioquia y UNAL - Universidad Nacional de Colombia (2012). Valoración económica, ecológica y sociocultural de bienes y servicios ambientales en la cuenca del río Grande. Medellín: Corantioquia y Universidad Nacional de Colombia, 544 p.

EPM-Empresas Públicas de Medellín (2005). Cincuenta años de hidrometeorología en Empresas Públicas de Medellín. Revista Hidrometeorológica, vol. 1, n. 1, 150 p.

Hargreaves, George y Samani, Zohrab (1985). Reference crop evapotranspiration from temperature. En: Applied Engineering in Agriculture, vol. 1, n. ${ }^{\circ}$ 2, p. 96-99.

Kok, Kasper; Verburg, Peter y Veldkamp, Antonie (2004). Integrated assessment of the land system: the future of land use. En: Land Use Policy, n. ${ }^{\circ} 24$, p. 517-520.

Lam, Quang Dung; Schmalz, Britta y Fohrer, Nicola (2011). The impact of agricultural Best Management Practices on water quality in a North German lowland catchment. En: Environmental Monitoring and Assessment, vol. 183, n. ${ }^{\circ} 1-4$, p. 351-379.

Lambin, Eric; Rounsevell, Mark y Geist, Helmut (2000). Are agricultural land-use models able to predict changes in land-use intensity? En: Agriculture, Ecosystems y Environment, vol. 82, n. $1-3$, p. $321-331$. 
León, Fernando y Renner, Isabel (2010). Conservation of water sources in Moyobamba: A brief review of the first experience in payments for environmental services in Peru. En: Mountain Forum Bulletin, vol. 10, n. ${ }^{\circ}$ 1, p. 85-86.

Moriasi, Daniel; Arnold, Jefrey; Van Liew, Michael; Bingner, Ronald; Harmel, Daren y Veith, Tamie (2007). Model evaluation guidelines for systematic quantification of accuracy in watershed simulations. En: Transactions of the Asabe, vol. 50, n. ${ }^{\circ}$ 3, p. 885-900.

Neitsch, Susan; Arnold, Jeff y Williams, Jimmy (2011). Soil and water assessment tool theoretical documentation version 2009. Texas: Texas Water Resources Institute, 618 p.

Quintero, Marcela; Estrada, Rubén Darío y García, James (2006). Modelo de optimización para evaluación ex ante de alternativas productivas y cuantificación de externalidades en cuencas andinas: modelo de evaluación económica, social y ambiental de usos de la tierra (Ecosaut). Lima: CIP, $76 \mathrm{p}$.

Quintero, Marcela; Wunder, Sven y Estrada, Rubén Darío (2009). For services rendered? Modeling hydrology and livelihoods in Andean payments for environmental services schemes. En: Forest Ecology and Management, vol. 258, n. ${ }^{\circ}$ 9, p. 1871-1880.

Ritchie, Joe (1972). Model for predicting evaporation from a row crop with incomplete cover. En: Water Resources Research, vol. 8, n. ${ }^{\circ}$ 5, p. 1204-1213.

Utgra-Unión Temporal para la Gestión del Riesgo Ambiental (2014). Formulación de indicadores para evaluar y monitorear las dinámicas de los agroecosistemas, las racionalidades y estrategias productivas de los agricultores en escenarios de cambio climático. Medellín: Utgra.

Yevenes, Mariela y Mannaerts, Chris (2011). Seasonal and land use impacts on the nitrate budget and export of a mesoscale catchment in Southern Portugal. En: Agricultural Water Management, vol. 102, n. ${ }^{\circ}$, p. 54-65. 